\title{
ACCURATE DIFFERENCE METHODS FOR NONLINEAR TWO-POINT BOUNDARY VALUE PROBLEMS*
}

\author{
HERBERT B. KELLER†
}

\begin{abstract}
We show that each isolated solution, $y(t)$, of the general nonlinear two-point boundary value problem $\left(^{*}\right): y^{\prime}=f(t, y), a<t<b, g(y(a), y(b))=0$ can be approximated by the (box) difference scheme $\left({ }^{* *}\right):\left[u_{j}-u_{j-1}\right] / h_{j}=f\left(t_{j-1 / 2},\left[u_{j}+u_{j-1}\right] / 2\right), 1 \leqq j \leqq J, g\left(u_{0}, u_{J}\right)=0$. For $h=\max _{1 \leqq j \leqq J} h_{j}$ sufficiently small, the difference equations $\left({ }^{* *}\right)$ are shown to have a unique solution $\left\{u_{j}\right\}_{0}^{J}$ in some sphere about $\left\{y\left(t_{j}\right)\right\}_{0}^{J}$, and it can be computed by Newton's method which converges quadratically. If $y(t)$ is sufficiently smooth, then the error has an asymptotic expansion of the form $u_{j}-y\left(t_{j}\right)=\sum_{v=1}^{m} h^{2 v} e_{v}\left(t_{j}\right)$ $+O\left(h^{2 m+2}\right)$, so that Richardson extrapolation is justified.

The coefficient matrices of the linear systems to be solved in applying Newton's method are of order $n(J+1)$ when $y(t) \in \mathbb{R}^{n}$. For separated endpoint boundary conditions: $g_{1}(y(a))=0, g_{2}(y(b))=0$ with $\operatorname{dim} g_{1}=p, \operatorname{dim} g_{2}=q$ and $p+q=n$, the coefficient matrices have the special block tridiagonal form $A \equiv\left[B_{j}, A_{j}, C_{j}\right]$ in which the $n \times n$ matrices $B_{j}\left(C_{j}\right)$ have their last $q$ (first $p$ ) rows null. Block elimination and band elimination without destroying the zero pattern are shown to be valid. The numerical scheme is very efficient, as a worked out example illustrates.
\end{abstract}

1. Introduction. We study the application of the centered Euler or box scheme to very general nonlinear two-point boundary value problems of the form

$$
\begin{gathered}
N y(t) \equiv y^{\prime}(t)-f(t, y(t))=0, \quad a \leqq t \leqq b ; \\
g(y(a), y(b))=0 .
\end{gathered}
$$

Such problems may, of course, have nonunique solutions. But we show that for each isolated solution of (1.1), the difference scheme has, for a sufficiently fine net, a unique solution in some tube about the isolated solution; the numerical solution can be computed by Newton's method with quadratic convergence; there is an asymptotic error expansion proceeding in powers of $h^{2}$ so that Richardson extrapolation is valid and yields two orders of magnitude improvement per application. When the boundary conditions are of the separated endpoint type, then the Newton iterates can be computed by a block elimination procedure which is very efficient. The net employed can be nonuniform, while the solution and "coefficients" in the equation need only be piecewise smooth. With very little effort, our theory is extended to include multipoint boundary conditions of the form

$$
g\left(y\left(\tau_{1}\right), y\left(\tau_{2}\right), \cdots, y\left(\tau_{N}\right)\right)=0, \quad a \leqq \tau_{1}<\tau_{2}<\cdots<\tau_{N} \leqq b .
$$

These results generalize a previous study of accurate difference schemes for linear multipoint boundary value problems [3] to the nonlinear case. Indeed, the basic stability result from this earlier study is crucial in the present analysis. Somewhat similar results for nonlinear two-point boundary value problems have been given in [4, pp. 96-102], but only for linear boundary conditions under quite

\footnotetext{
* Received by the editors November 9, 1972, and in revised form March 1, 1973.

+ Applied Mathematics, Firestone Laboratories, California Institute of Technology, Pasadena, California 91109. This work was supported by the Atomic Energy Commission under Contract AT(04-3)-767, Project Agreement no. 12.
} 
unnatural restrictions. The present basic requirement that the solution of (1.1) be isolated is close to the minimal conditions to be expected.

The present theory has been extended to very general difference schemes which are defined in terms of one-step and multistep schemes for initial value problems. In fact any numerical scheme which is stable and consistent for initial value problems can be shown to yield a difference scheme to which our main theorem (trivially modified) applies. These results will be published in the future [10]. One particularly significant such extension due to R. Weiss [11] employs implicit Runge-Kutta schemes which with the appropriate $m$-point Lobatto quadrature points have $O\left(h^{2 m-2}\right)$ accuracy. Weiss further shows the equivalence of the difference schemes with appropriate collocation methods using piecewise polynomials. Recently H.-O. Kreiss [6] has developed a very general and quite complete theory of difference methods for linear boundary value problems. It is rather clear that his work can be extended to nonlinear boundary value problems by essentially the same techniques used in the present work.

$A$ solution $y(t) \in \mathbb{R}^{n}$ of (1.1) is said to be isolated if and only if the linearized problem

$$
\begin{aligned}
L[y] \phi(t) \equiv & \phi^{\prime}-A(t) \phi(t)=0, \quad a \leqq t \leqq b ; \\
& B_{a} \phi(a)+B_{b} \phi(b)=0,
\end{aligned}
$$

with the $n \times n$ matrices

$$
A(t) \equiv \partial f(t, y(t)) / \partial y, \quad B_{a} \equiv \partial g(y(a), y(b)) / \partial y(a), \quad B_{b} \equiv \partial g(y(a), y(b)) / \partial y(b)
$$

has only the trivial solution $\phi(t) \equiv 0$. It is more or less well known that an isolated solution is "locally unique"; that is, no other solution exists in some tube about the isolated solution.

The difference scheme is employed on any (nonuniform) net $\left\{t_{j}\right\}_{0}^{J}$ with

$$
\begin{array}{ll}
t_{0}=a ; & t_{j}=t_{j-1}+h_{j}, \quad 1 \leqq j \leqq J ; \\
t_{J}=b ; & h \equiv \max h_{j} \leqq \lambda \min h_{j} .
\end{array}
$$

Here $\lambda$ is a uniform bound on the ratio of maximum to minimum spacing for all families of nets we consider. Applied to (1.1), the box scheme (or centered Euler scheme) is

$$
\begin{gathered}
N_{h} u_{j} \equiv \frac{1}{h_{j}}\left[u_{j}-u_{j-1}\right]-f\left(t_{j-1 / 2}, \frac{1}{2}\left[u_{j}+u_{j-1}\right]\right)=0, \quad 1 \leqq j \leqq J, \\
g\left(u_{0}, u_{J}\right)=0
\end{gathered}
$$

Our basic results may be stated as the following theorem.

MAIN TheOREM. Let (1.1) have an isolated solution $y(t) \in C_{4}[a, b]$. Let $f(t, z) \in C_{3}\left\{[a, b] \times S_{\rho}[y(t)]\right\}$, where $S_{\rho}[y(t)] \equiv\left\{z \mid z \in \mathbb{R}^{n},\|z-y(t)\| \leqq \rho\right\} \subset \mathbb{R}^{n}$ and $g(v, w) \in C_{2}\left\{S_{\rho}[y(a)] \times S_{\rho}[y(b)]\right\}$. Define the sphere $S_{\rho}\left\{y\left(t_{j}\right)\right\} \equiv\left\{v_{0}, \cdots, v_{J} \mid v_{j} \in S_{\rho}\right.$ $\left.\cdot\left[y\left(t_{j}\right)\right], 0 \leqq j \leqq J\right\} \subset \mathbb{R}^{n(J+1)}$. Then for some $\rho$ and $h_{0}$ sufficiently small, all nets (1.3a) with $h \leqq h_{0}$ are such that:

(i) The difference equations (1.3) have a unique solution $\left\{u_{j}\right\} \in S_{\rho}\left\{y\left(t_{j}\right)\right\}$. 
(ii) The difference solution can be computed by Newton's method, (3.5), which converges quadratically for any initial iterate $\left\{u_{j}^{(0)}\right\} \in S_{\rho_{1}}\left\{y\left(t_{j}\right)\right\}$, provided $\rho_{1}$ and $\rho_{1} / h$ are sufficiently small.

(iii) $\left\|u_{j}-y\left(t_{j}\right)\right\|=O\left(h^{2}\right), 0 \leqq j \leqq J$.

(iv) If $f(t, z)$ and $y(t)$ are sufficiently smooth, then there exist $e_{v}(t)$ such that

$$
u_{j}^{(k)}-y\left(t_{k, j}\right)=\sum_{v=1}^{m}\left(\frac{h_{k}}{2}\right)^{2 v} e_{v}\left(t_{k, j}\right)+O\left(h_{k}^{2 m+2}\right), \quad 0 \leqq j \leqq J_{k}, \quad k=0,1, \cdots,
$$

for all nets $\left\{t_{k, j}\right\}$ satisfying (4.10). Here $u_{j}^{(k)}$ is the numerical solution on the $k$-th net.

In $\S 2$ we present some results from [3] which are valid for linear two-point boundary value problems; this is the basic stability theory we employ. Parts (i) and (ii) of the main theorem are proven in $\S 3$ and parts (iii) and (iv) are proven in $\S 4$. In $\S 5$ we show that for separated endpoint boundary conditions, i.e., for $g_{1}(y(a))=0, g_{2}(y(b))=0$, the linearized equations which arise in Newton's method can be solved by very efficient block-elimination or band-elimination procedures. Some practical observations on the use of Newton's method in conjunction with Richardson extrapolation are given in $\S 6$. A worked out example is also reported there. Our method has been used to solve nonlinear systems with as many as $n=120$ equations, and this work is reported elsewhere [7]. In the Appendix we indicate how the basic results can be extended to apply to nonlinear multipoint boundary conditions.

It should be observed that all of our results apply equally well to the trapezoidal rule:

$$
\frac{1}{h_{j}}\left[u_{j}-u_{j-1}\right]-\frac{1}{2}\left[f\left(t_{j-1}, u_{j-1}\right)+f\left(t_{j}, u_{j}\right)\right]=0 .
$$

We have used centered Euler as in (1.3b) as it is frequently more efficient.

2. Summary of results for linear problems. We summarize here some of the results of [3] specialized for linear two-point boundary value problems of the form

$$
\begin{gathered}
L v \equiv v^{\prime}-A(t) v=g(t), \\
B_{a} v(a)+B_{b} v(b)=\beta .
\end{gathered}
$$

It is well known and easily demonstrated (see [3]), that (2.1) has a unique solution for each $g(t) \in C[a, b]$ and each $\beta \in \mathbb{R}^{n}$ if and only if the $n \times n$ matrix

$$
Q \equiv B_{a} Y(a, \tau)+B_{b} Y(b, \tau)
$$

is nonsingular. Here $Y(t, \tau)$ is the fundamental solution matrix of $(2.1 \mathrm{a})$ defined for any $\tau \in[a, b]$ by

$$
Y^{\prime}=A(t) Y, \quad a \leqq t \leqq b
$$

$$
Y(\tau)=I
$$

In most applications we employ $\tau=a$ (or $\tau=b)$. 
The centered Euler or box scheme for (2.1) on the net (1.3a) is

(2.4a) $L_{h} v_{j} \equiv h_{j}^{-1}\left(v_{j}-v_{j-1}\right)-A\left(t_{j-1 / 2}\right) \frac{1}{2}\left(v_{j}+v_{j-1}\right)=g\left(t_{j-1 / 2}\right), \quad 1 \leqq j \leqq J ;$

$$
B_{a} v_{0}+B_{b} v_{J}=\beta \text {. }
$$

We combine Lemmas 3.1-3.3 of [3] to state the following.

LEMMA 2.1. Let $B_{a}, B_{b}$ and $A(t) \in C[a, b]$ be such that (2.1) has a unique solution. Then there exist constants $h_{0}, K_{1}, K_{2}$ such that for every net (1.3a) with $h \leqq h_{0}$ the linear difference equations (2.4) have a unique solution which is bounded by

$$
\left\|v_{j}\right\| \leqq K_{1}\|\beta\|+K_{2} \max _{1 \leqq i \leqq J}\left\|g\left(t_{i-1 / 2}\right)\right\|, \quad 0 \leqq j \leqq J .
$$

The linear system (2.4) can be written in the matrix-vector form

$$
\mathbb{L} V=G
$$

by introducing the $n \times n$ matrices

$$
L_{j} \equiv h_{j}^{-1} I+\frac{1}{2} A\left(t_{j-1 / 2}\right), \quad R_{j} \equiv h_{j}^{-1} I-\frac{1}{2} A\left(t_{j-1 / 2}\right),
$$

and the $n(J+1)$ order matrix and vectors

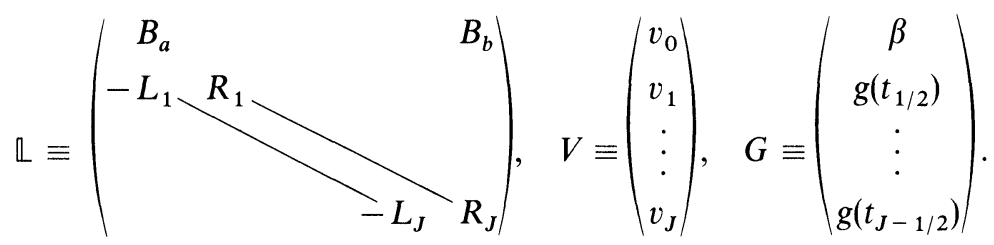

Lemma 2.1 clearly implies that $\mathbb{L}$ is nonsingular for all nets (1.3a) with $h \leqq h_{0}$. To get a bound on $\left\|\mathbb{L}^{-1}\right\|$, we use the operator norm induced by the vector norm on $V \in \mathbb{R}^{n(J+1)}$ given by

$$
\|V\| \equiv \max _{0 \leqq j \leqq J}\left\|v_{j}\right\|,
$$

where $\|v\|$ is any vector norm on $v \in \mathbb{R}^{n}$. Then (2.5) implies

$$
\left\|\mathbb{L}^{-1}\right\| \leqq \max \left(K_{1}, K_{2}\right) \equiv K_{0} .
$$

This furnishes a bound which is uniform on the family of matrices $\mathbb{L}$ defined for all nets (1.3a) with $h \leqq h_{0}$.

3. Solution of the difference equations. We first prove part (i) of the main theorem by means of contracting maps. Then we examine Newton's method. Write (1.3) in the vector form

$$
\Phi(U)=0
$$

$$
U \equiv\left(\begin{array}{c}
u_{0} \\
u_{1} \\
\vdots \\
u_{J}
\end{array}\right)
$$




$$
\Phi(U)=\left(\begin{array}{c}
g\left(u_{0}, u_{J}\right) \\
N_{h} u_{1} \\
\vdots \\
N_{h} u_{J}
\end{array}\right) .
$$

Since $\mathbb{L}$ of (2.6) is nonsingular, employing (1.2c), we can write (3.1a) in the equivalent form

$$
U=U-\mathbb{L}^{-1} \Phi(U) \equiv \Psi(U) .
$$

For any $V, W \in S_{\rho}\left\{y\left(t_{j}\right)\right\}$, we have

$$
\begin{aligned}
\Psi(V)-\Psi(W) & =[V-W]-\mathbb{L}^{-1}[\Phi(V)-\Phi(W)] \\
& =\mathbb{L}^{-1}[\mathbb{L}-\partial \widetilde{\Phi}(V, W) / \partial U][V-W],
\end{aligned}
$$

where

$$
\frac{\partial \tilde{\Phi}(V, W)}{\partial U}=\int_{0}^{1} \frac{\partial \Phi}{\partial U}(s V+[1-s] W) d s
$$

Here we have used the convexity of $S_{\rho}\left\{y\left(t_{j}\right)\right\}$ and the continuous differentiability of $f(t, z)$ and $g(v, w)$. From (1.2c) we recall that $A\left(t_{j-1 / 2}\right)=f_{y}\left(t_{j-1 / 2}, y\left(t_{j-1 / 2}\right)\right)$. Now introduce $\widehat{A}\left(t_{j-1 / 2}\right) \equiv f_{y}\left(t_{j-1 / 2}, \frac{1}{2}\left[y\left(t_{j}\right)+y\left(t_{j-1}\right)\right]\right)$ and denote by $\widehat{\mathbb{L}}$ the matrix of $(2.6 \mathrm{~b}, \mathrm{c})$ with $A\left(t_{j-1 / 2}\right)$ replaced by $\widehat{A}\left(t_{j-1 / 2}\right)$ for $1 \leqq j \leqq J$. Then

$$
\begin{aligned}
\left\|\hat{\mathbb{L}}-\frac{\partial \tilde{\Phi}}{\partial U}\right\| & \leqq\|\mathbb{L}-\hat{\mathbb{L}}\|+\left\|\hat{\mathbb{L}}-\frac{\partial \tilde{\Phi}}{\partial U}\right\| . \\
& \leqq K \max _{j}\left\|y\left(t_{j-1 / 2}\right)-\frac{1}{2}\left[y\left(t_{j}\right)+y\left(t_{j-1}\right)\right]\right\|+K \rho,
\end{aligned}
$$

where $K$ is the maximum of the Lipschitz constants for $f_{v}(t, z), g_{v}(v, w), g_{w}(v, w)$ with respect to $z, v, w$. If, as is implied by the hypothesis, $y(t) \in C_{4}[a, b]$, then clearly for some $M_{0}>0$,

$$
\|\mathbb{L}-\partial \tilde{\Phi} / \partial U\| \leqq K\left(M_{0} h^{2}+\rho\right) .
$$

Combined with (2.8), this result in (3.3a) yields

$$
\|\Psi(V)-\Psi(W)\| \leqq \alpha\|V-W\|, \quad \alpha \equiv K_{0} K\left(M_{0} h^{2}+\rho\right)<1,
$$

for all $h \leqq h_{0}$, provided $h_{0}$ and $\rho$ are sufficiently small.

The "center" of the sphere $S_{\rho}\left\{y\left(t_{j}\right)\right\}$ is denoted by $Y \equiv\left(y\left(t_{0}\right), \cdots, y\left(t_{J}\right)\right)^{T}$. Then $\Phi(Y)$ can be estimated from (3.1c), (1.1) and (3.1b) by

$$
\begin{aligned}
\|\Phi(Y)\| & =\max _{1 \leqq j \leqq J}\left\|N_{h} y\left(t_{j}\right)\right\| \\
& =\max _{1 \leqq j \leqq J}\left\|N_{h} y\left(t_{j}\right)-N y\left(t_{j-1 / 2}\right)\right\| \\
& \leqq M_{1} h^{2} .
\end{aligned}
$$


This is just a bound on the local truncation errors which, with (3.2) and (2.8), now give

$$
\|Y-\Psi(Y)\| \leqq K_{0} M_{1} h^{2} \leqq(1-\alpha) \rho,
$$

for all $h \leqq h_{0}$, provided $h_{0}$ is sufficiently small.

From (3.4a, b) it follows that $\Psi(U)$ takes $S_{\rho}\left\{y\left(t_{j}\right)\right\}$ into itself and is contracting there. Thus (3.2) has a unique solution in $S_{\rho}\left\{y\left(t_{j}\right)\right\}$, and part (i) of the main theorem is established. We point out that the iteration scheme suggested by (3.2) is not practical since $\mathbb{L}$ is, of course, unknown. In many applications, Newton's method is extremely effective, so we present a theoretical justification for it showing the quadratic convergence.

Specifically, we define the sequence of Newton iterates $U^{v}$ by

$$
\begin{gathered}
U^{0} \in S_{\rho_{1}}\left\{y\left(t_{j}\right)\right\} ; \\
\frac{\partial \Phi\left(U^{v}\right)}{\partial U}\left[U^{v+1}-U^{v}\right]=-\Phi\left(U^{v}\right), \quad v=0,1,2, \cdots .
\end{gathered}
$$

Note, for any $U \in S_{\rho}\left\{y\left(t_{j}\right)\right\}$, that as in (3.3c), $\left\|\mathbb{L}^{-1}(\mathbb{L}-\partial \Phi(U) / \partial U)\right\| \leqq \alpha<1$. Then since $\partial \Phi(U) / \partial U=\mathbb{L}\left[I-\mathbb{L}^{-1}(\mathbb{L}-\partial \Phi(U) / \partial U)\right]$, it follows from the Banach lemma that $\partial \Phi(U) / \partial U$ is nonsingular for all $U \in S_{\rho}\left\{y\left(t_{j}\right)\right\}$. Further, this lemma and (2.8) imply

$$
\left\|\left(\frac{\partial \Phi(U)}{\partial U}\right)^{-1}\right\| \leqq \frac{K_{0}}{1-\alpha} .
$$

With the same Lipschitz constant $K$ used in (3.3c), we have for all $U, V \in S_{\rho}\left\{y\left(t_{j}\right)\right\}$,

$$
\|\partial \Phi(U) / \partial U-\partial \Phi(V) / \partial V\| \leqq K\|U-V\| \text {. }
$$

The initial error in satisfying (3.1a) can be estimated as

$$
\begin{aligned}
\left\|\Phi\left(U^{0}\right)\right\| & \leqq\|\Phi(Y)\|+\left\|\Phi\left(U^{0}\right)-\Phi(Y)\right\| \\
& \leqq M_{1} h^{2}+\|\partial \widetilde{\Phi} / \partial U\| \cdot\left\|U^{0}-Y\right\| \\
& \leqq M_{1} h^{2}+2(\lambda / h+C) \rho_{1},
\end{aligned}
$$

where $C$ is a bound on the norms of $g_{v}(v, w), g_{w}(v, w)$ and $\frac{1}{2} f_{u}(t, u)$, and $\lambda$ is defined in (1.3a). The quadratic convergence of Newton's method now follows from (3.6) in standard fashion provided $h, \rho_{1}$ and $\rho_{1} / h$ are sufficiently small; see, for example, [5] or [8].

In practice, the basic problem is, as usual, to find any appropriate initial iterate $U^{0}$ which is within a distance $\rho_{1}$ from $Y$. We discuss some of the practical considerations in $\S \S 5$ and 6 . But it is important to note that as $h$ is reduced, $\rho_{1}$ must also be reduced in order that $\left\|\Phi\left(U^{0}\right)\right\|$ be sufficiently small. The implication is that we must be able to guess at an $O(h)$ accurate solution to get our Newton scheme to converge. Once this is done for any fixed $h$, we shall see that we can then easily compute solutions accurate to $O\left(h^{2 m}\right)$ for some $m=1,2, \cdots$.

4. Error estimates. To establish part (iii) of the main theorem, let $v_{j} \equiv u_{j}-y\left(t_{j}\right)$, where $\left\{u_{j}\right\}$ is the solution of (1.3) and $y(t)$ is the solution of (1.1). 
Then since $N_{h} u_{j}=N_{h}\left[y\left(t_{j}\right)+v_{j}\right]=0$, the mean value theorem yields

$$
\tilde{L}_{h} v_{j}=-N_{h} y\left(t_{j}\right) \equiv \tau_{j}[y], \quad 1 \leqq j \leqq J .
$$

Similarly, (1.1b) and (1.3c) imply

$$
\widetilde{B}_{a} v_{0}+\widetilde{B}_{b} v_{J}=0
$$

Here $\tilde{L}_{h}$ is given by $(2.4 \mathrm{a})$ with $A\left(t_{j-1 / 2}\right)$ replaced by

$$
\tilde{A}_{j-1 / 2} \equiv \int_{0}^{1} f_{y}\left(t_{j-1 / 2}, \frac{1}{2}\left[y\left(t_{j}\right)+y\left(t_{j-1}\right)\right]+\frac{s}{2}\left[v_{j}+v_{j-1}\right]\right) d s
$$

and

$$
\begin{aligned}
& \widetilde{B}_{a} \equiv \int_{0}^{1} g_{y(a)}\left(y(a)+s v_{0}, y(b)+s v_{J}\right) d s, \\
& \widetilde{B}_{b} \equiv \int_{b}^{1} g_{y(b)}\left(y(a)+s v_{0}, y(b)+s v_{J}\right) d s .
\end{aligned}
$$

A standard Taylor expansion yields, with (1.1a),

$$
\begin{aligned}
\left\|\tau_{j}[y]\right\| & =\left\|N_{y}\left(t_{j-1 / 2}\right)-N_{h} y\left(t_{j}\right)\right\| & \\
& \leqq M_{1} h^{2}, & 1 \leqq j \leqq J .
\end{aligned}
$$

The system $(4.1 \mathrm{a}, \mathrm{b})$ can now be written as

$$
\begin{gathered}
\tilde{\mathbb{L}} V=T, \\
V \equiv\left(\begin{array}{c}
v_{0} \\
v_{1} \\
\vdots \\
v_{J}
\end{array}\right), \quad T \equiv\left(\begin{array}{c}
0 \\
\tau_{1}[y] \\
\vdots \\
\tau_{J}[y]
\end{array}\right),
\end{gathered}
$$

where $\widetilde{\mathbb{L}}$ is defined by $(2.6 \mathrm{~b}, \mathrm{c})$ with $A_{j-1 / 2}, B_{a}$ and $B_{b}$ replaced by $\widetilde{A}_{j-1 / 2}, \widetilde{B}_{a}$ and $\widetilde{B}_{b}$, respectively, of (4.2). It follows that

$$
\|\mathbb{L}-\tilde{\mathbb{L}}\| \leqq \frac{K}{2}\|V\|,
$$

where $K$ is the Lipschitz constant previously introduced. Since $\mathbb{L}$ has a uniformly bounded inverse, as in (2.8), write (4.4a) as $\mathbb{L} V=(\mathbb{L}-\widetilde{\mathbb{L}}) V+T$ and use (4.5) and (4.3) to deduce

$$
\|V\| \leqq \frac{K_{0} K}{2}\|V\|^{2}+K_{0} M_{1} h^{2}
$$

The scalar inequality $x \leqq \alpha x^{2}+\beta$, with $\alpha>0$ and $4 \alpha \beta<1$, implies that either $x \leqq x_{-}$or $x \geqq x_{+}$where $x_{ \pm}=(1 \pm \sqrt{1-4 \alpha \beta}) / 2 \alpha$. To apply this result in (4.6), let us require $h_{0}$ to be so small that

$$
2 K_{0}^{2} K M_{1} h^{2}<\frac{1}{2} \text { for all } h \leqq h_{0} .
$$


Then recalling $\|V\|=\|U-Y\| \leqq \rho$, we also require

$$
\rho<\left(1+\sqrt{1-2 K_{0}^{2} K M_{1} h_{0}^{2}}\right) / K_{0} K
$$

and it follows that

$$
\begin{aligned}
\|V\| & \leqq \\
& \leqq \sqrt{2} K_{0} M_{1} h^{2} .
\end{aligned}
$$

Thus part (iii) is proven.

The asymptotic error expansions in part (iv) of the main theorem follow by showing that the coefficient functions, $e_{v}(t)$, can be defined recursively, with $e^{0}(t)$ $\equiv y(t)$, by linear two-point boundary value problems of the form

$$
\begin{aligned}
\frac{d}{d t} e^{v}(t)-A(t) e^{v}(t) & =\Theta_{v}(t), & v & =1,2, \cdots, \\
B_{a} e^{v}(a)+B_{b} e^{v}(b) & =\gamma_{v}, & v & =1,2, \cdots .
\end{aligned}
$$

In view of (1.2) and the fact that $y(t)$ is an isolated solution of (1.1), it follows that (4.9) has a unique solution for each, say continuous, $\Theta_{v}(t)$ and bounded $\gamma_{v}$. We must, of course, specify how this inhomogeneous data is determined. To allow nonuniform spacing, we consider only sequences of nets $\left\{t_{k, j}\right\}, k=0,1, \cdots$, of the form

$$
\begin{aligned}
& t_{k, 0}=a ; \quad t_{k, j}=t_{k, j-1}+h_{k, j}, \quad 1 \leqq j \leqq J_{k} ; \\
& t_{k, J_{k}}=b ; \quad h_{k} \equiv \max _{j} h_{k, j} \leqq \lambda \min _{j} h_{k, j},
\end{aligned}
$$

where for some piecewise $C^{\infty}$-function $\phi(t)$, with jump discontinuities at most confined to the points $\left\{t_{0, j}\right\}_{0}^{J_{0}}$ of the initial net, the spacings are such that

$$
h_{k, j}=h_{k} \phi\left(t_{k, j-1 / 2}\right) \equiv h_{k} \phi\left(\frac{1}{2}\left[t_{k, j}+t_{k, j-1}\right]\right) ; \quad h_{k+1}<h_{k} .
$$

The proof and the "derivation" of the $\Theta_{v}(t)$ and $\gamma_{v}$ proceed essentially by induction and are by now standard. Indeed, the corresponding development for initial value problems as in [1] contains all the basic ideas and yields the form of the $\Theta_{v}(t)$. The modifications required for variable net spacing are contained in [3]. The boundary conditions are easily treated using expansions analogous to those for the differential equations. Thus the proof of the main theorem is concluded.

5. Separated endpoints and block elimination. Perhaps the most important practical observation in applying (1.3) with Newton's method (3.5) to solve the difference equations is the fact that many problems have separated endpoint boundary conditions. That is, $g(v, w)$ in $(1.1 \mathrm{~b})$ can be written as

$$
g(v, w) \equiv\left(\begin{array}{l}
g_{1}(v) \\
g_{2}(w)
\end{array}\right),
$$

where, say, $g_{1}(v)$ is a $p$-vector and $g_{2}(w)$ is a $q \equiv(n-p)$-vector. Then from $(1.2 \mathrm{c})$,

$$
B_{a} \equiv\left(\begin{array}{c}
M_{a} \\
\mathbf{0}_{q \times n}
\end{array}\right), \quad B_{b} \equiv\left(\begin{array}{c}
\mathbf{0}_{p \times n} \\
M_{b}
\end{array}\right), \quad\left\{\begin{array}{l}
M_{a} \equiv \frac{\partial g_{1}(y(a))}{\partial y} \text { is } p \times n \\
M_{b} \equiv \frac{\partial g_{2}(y(b))}{\partial y} \text { is } q \times n
\end{array}\right.
$$


It is assumed that rank $M_{a}=p$ and rank $M_{b}=q$ for all arguments to be employed. This insures $p$ independent constraints at $x=a$ and $q$ independent constraints at $x=b$. If we write the first $p$-boundary conditions first and the last $q$-boundary conditions last, then $\Phi(U)$ is not as defined in (3.1c), but instead is

$$
\Phi(U) \equiv\left(\begin{array}{c}
g_{1}\left(u_{0}\right) \\
N_{h} u_{1} \\
\vdots \\
N_{h} u_{J} \\
g_{2}\left(u_{J}\right)
\end{array}\right)
$$

The linear system for Newton's method applied to solve $\Phi(U)=0$ now has the form

$$
\mathbb{A}^{v}\left[U^{v+1}-U^{v}\right]=-\Phi\left(U^{v}\right)
$$

where

$$
\begin{aligned}
& \mathbb{A}^{v} \equiv\left(\begin{array}{ccc}
M_{a}^{v} & & \\
-L_{1}^{v} & R_{1}^{v}-L_{J}^{v} & 0 \\
0 & R_{J}^{v} \\
& & M_{b}^{v}
\end{array}\right), \\
& \left\{\begin{array}{l}
L_{j}^{v} \equiv h_{j}^{-1} I+\frac{1}{2} \frac{\partial f}{\partial y}\left(t_{j-1 / 2}, \frac{1}{2}\left[u_{j}^{v}+u_{j-1}^{v}\right]\right) \\
R_{j}^{v} \equiv h_{j}^{-1} I-\frac{1}{2} \frac{\partial f}{\partial y}\left(t_{j-1 / 2}, \frac{1}{2}\left[u_{j}^{v}+u_{j-1}^{v}\right]\right) \\
M_{a}^{v} \equiv \frac{\partial g_{1}\left(u_{0}^{v}\right)}{\partial y}, M_{b}^{v} \equiv \frac{\partial g_{2}\left(u_{J}^{v}\right)}{\partial y} .
\end{array}\right.
\end{aligned}
$$

The $\mathbb{A}^{v}$ are nonsingular when part (ii) of the main theorem holds, as they are obtained from row interchanges of the (nonsingular) $\partial \Phi\left(U^{v}\right) / \partial U$ introduced in $\S 3$. Clearly each $A^{v}$ is a band matrix of bandwidth at most $2 n$ so that efficient band- or block-elimination methods can be used to solve (5.3a). In particular, efficient elimination schemes can be devised by writing the $A^{v}$ in block tridiagonal form

$$
\mathbb{A}^{v} \equiv\left[B_{i}^{v}, A_{i}^{v}, C_{i}^{v}\right], \quad 0 \leqq i \leqq J,
$$

where the $A_{i}^{v}, B_{i}^{v}$ and $C_{i}^{v}$ are $n \times n$ matrices defined by equating the representations in (5.3b) and (5.4). For example, with $J=3$ the old and new representations are schematically represented as Fig. 1 . The solid squares are the $\left(-L_{j}^{v}\right)$ and $\left(R_{j}^{v}\right)$, the solid rectangles are $M_{a}^{v}$ and $M_{b}^{v}$. The dotted lines indicate the rows of zeros that are adjoined and how the $L_{j}^{v}$ and $R_{j}^{v}$ are partitioned to form the $A_{i}^{v}, B_{i}^{v}$ and $C_{i}^{v}$. Thus each $B_{i}^{v}$ has its final $q$ rows all zeros and each $C_{i}^{v}$ has its first $p$ rows all zeros. It should be recalled that if the original problem (1.1) is linear with separated endpoint conditions, then our difference equations written in the form (5.2) already have a coefficient matrix of the same form as the $\mathbb{A}^{v}$ above. 


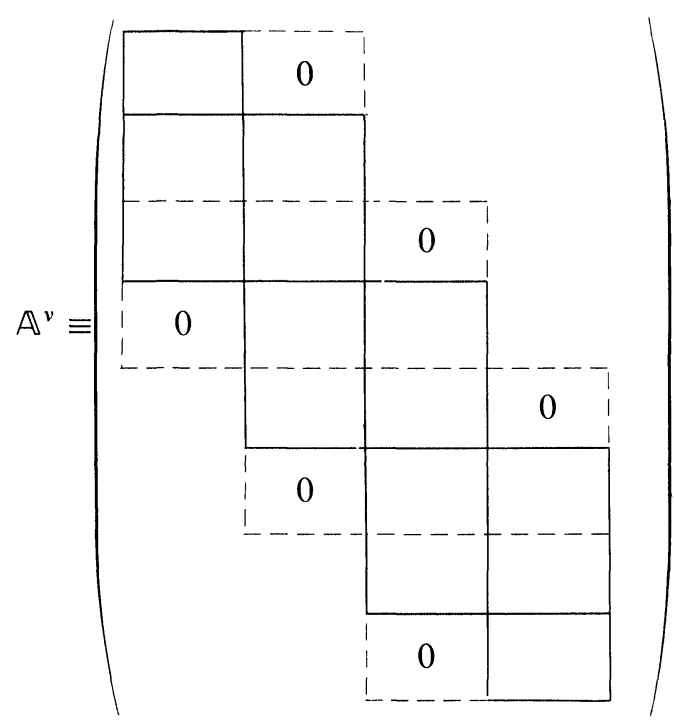

Fig. 1

We now examine methods for solving linear systems with coefficient matrices of the form (5.4) in which the submatrices have the zero elements indicated above. To simplify notation we drop the iteration index $v$. Thus we consider nonsingular linear systems of order $n(J+1)$ which have the form

$$
A \mathbf{X}=\mathbf{F},
$$

where

$$
\begin{array}{ll}
\mathbb{A} \equiv\left[B_{j}, A_{j}, C_{j}\right], & 0 \leqq j \leqq J \\
\mathbf{X}^{T} \equiv\left(\mathbf{x}_{0}, \cdots, \mathbf{x}_{J}\right) ; & \mathbf{F}^{T} \equiv\left(\mathbf{f}_{0}, \cdots, \mathbf{f}_{J}\right),
\end{array}
$$

and the $n \times n$ submatrices have the zero rows indicated in

$$
\left.B_{j} \equiv\left(\begin{array}{cccc}
x & x & \cdots & x \\
\vdots & & & \vdots \\
x & x & \cdots & x \\
0 & & & 0 \\
\vdots & & & \vdots \\
0 & & & 0
\end{array}\right), \quad C_{j} \equiv\left(\begin{array}{cccc}
0 & & & 0 \\
\vdots & & & \vdots \\
0 & & & 0 \\
x & x & \cdots & x \\
\vdots & & & \vdots \\
x & x & \cdots & x
\end{array}\right)\right\} q, \quad p+q=n
$$

To solve (5.4a), we seek factorizations of $A$ into the form

$$
\mathbb{A}=\mathbb{L} \mathbb{U} ; \quad \mathbb{L} \equiv\left[\beta_{j}, \delta_{j}, \mathbf{0}\right], \quad \mathbb{U} \equiv\left[\mathbf{0}, \alpha_{j}, \gamma_{j}\right], \quad 0 \leqq j \leqq J
$$

Here $\alpha_{j}, \beta_{j}, \gamma_{j}$ and $\mathbf{0}$ are $n \times n$ matrices, the latter having all zero elements, so that $\mathbb{L}$ and $\mathbb{U}$ are also block-tridiagonal, in fact block-lower and -upper triangular as well. The solution of $(5.4 \mathrm{a})$ is then equivalent to solving the two systems 


$$
\begin{aligned}
\mathbb{U} \mathbf{Y} & =\mathbf{F}, \\
\mathbb{U} \mathbf{X} & =\mathbf{Y} .
\end{aligned}
$$

The factorization in $(5.5 \mathrm{a})$ requires that

$$
\begin{aligned}
\delta_{0} \alpha_{0} & =A_{0} ; & & \\
\delta_{j} \alpha_{j} & =A_{j}-\beta_{j} \gamma_{j-1}, & & 1 \leqq j \leqq J \\
\beta_{j} \alpha_{j-1} & =B_{j}, & & 1 \leqq j \leqq J \\
\delta_{j} \gamma_{j} & =C_{j}, & & 0 \leqq j \leqq J-1 .
\end{aligned}
$$

The solution of $(5.5 \mathrm{~b}, \mathrm{c})$ is then obtained by solving the $n$th order systems

$$
\begin{aligned}
\delta_{0} \mathbf{y}_{0} & =\mathbf{f}_{0}, \\
\delta_{j} \mathbf{y}_{j} & =\mathbf{f}_{j}-\beta_{j} \mathbf{y}_{j-1}, \quad 1 \leqq j \leqq J ; \\
\alpha_{J} \mathbf{x}_{J} & =\mathbf{y}_{J}, \\
\alpha_{j} \mathbf{x}_{j} & =\mathbf{y}_{j}-\gamma_{j} \mathbf{x}_{j+1}, \quad J-1 \geqq j \geqq 0 .
\end{aligned}
$$

The factorization is not uniquely determined by $(5.6 \mathrm{a}-\mathrm{d})$, if it indeed exists at all. We distinguish four common choices each of which uniquely defines the factorization (assuming existence); these are

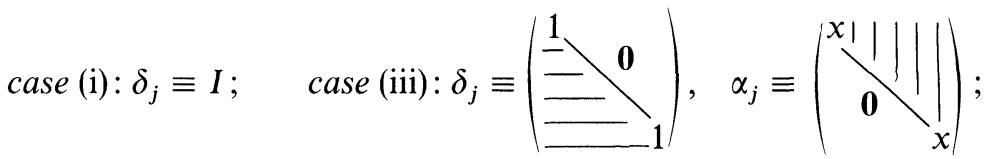

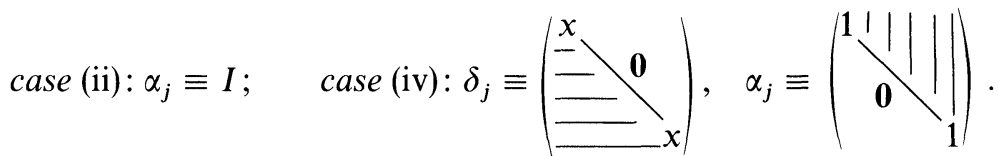

Cases (i) and (ii) are the standard block-tridiagonal factorization procedures, while cases (iii) and (iv) are standard band-factorization procedures or Gauss elimination accounting for zero elements. Assuming for the moment that they can all be carried out, it easily follows from $(5.6 \mathrm{c}, \mathrm{d})$ and $(5.4 \mathrm{c})$ that in all cases the $\beta_{j}$ have all zeros in the last $q$ rows, while in cases (i), (iii) and (iv) the $\gamma_{j}$ have all zeros in the first $p$ rows. In case (ii), the $\gamma_{j}$ may be full so that, as we shall see, this form of factorization should not be used. The operational counts for these schemes, accounting for all the null rows, are easily obtained by examining $(5.6 \mathrm{a}-\mathrm{f})$. They are summarized in Table 1 . In the totals and in $(5.6 \mathrm{e}, \mathrm{f})$, we have dropped lower order terms not proportional to $J$. These counts for cases (i) and (iii) have also been given by Varah [9], who also observes that case (i) is preferable to case (iii) when $p / n<0.38$ (based on cubic terms in the operational counts). Clearly, case (i) is always preferable to case (ii), while case (iii) is preferred to case (iv) when $p<q$.

The block-factorization cases (i) and (ii) can be performed with a restricted form of partial pivoting, which does not destroy the zero patterns in the $B_{j}$ and $C_{j}$. This can be used to insure that these procedures are valid in our present applications. The restricted partial pivoting merely amounts to reordering the difference 
TABLE 1

Operational counts for solving (5.4) using (5.6)

\begin{tabular}{|c|c|c|c|c|}
\hline To solve & Case (i) & Case (ii) & Case (iii) & Case (iv) \\
\hline$(5.6 a)$ & 0 & 0 & $n^{3} / 3$ & $n^{3} / 3$ \\
\hline$(5.6 \mathrm{~b})$ & $J n p q$ & $J n^{2} p$ & $J\left(n p q+n^{3} / 3-n / 3\right)$ & $J\left(n p q+n^{3} / 3-n / 3\right)$ \\
\hline$(5.6 \mathrm{c})$ & $J\left(n^{2} p+n^{3} / 3-n / 3\right)$ & 0 & $J p\left[n^{2}+n\right] / 2$ & $J p\left[n^{2}-n\right] / 2$ \\
\hline$(5.6 \mathrm{~d})$ & 0 & $\begin{array}{l}J\left(5 n^{3} / 6+n q^{2} / 2\right. \\
\quad+n p / 2-n / 3)\end{array}$ & $J h\left[q^{2}-q\right] / 2$ & $\operatorname{Jn}\left[q^{2}+q\right] / 2$ \\
\hline$(5.6 \mathrm{e})$ & $J n p$ & $J\left(n p+n^{2}\right)$ & $J\left(p n+\left[n^{2}-n\right] / 2\right)$ & $J\left(p n+\left[n^{2}+n\right] / 2\right)$ \\
\hline$(5.6 f)$ & $J\left(n q+n^{2}\right)$ & $J n^{2}$ & $J\left(q-n+\left[n^{2}+n\right] / 2\right)$ & $J\left(q n+\left[n^{2}-n\right] / 2\right)$ \\
\hline $\begin{array}{l}\text { Totals } \\
\text { (leading } \\
\text { orders) }\end{array}$ & $\begin{array}{l}J\left(4 n^{3} / 3-n q^{2}\right. \\
\left.\quad+2 n^{2}-n / 3\right)\end{array}$ & $\begin{array}{l}J\left(4 n^{3} / 3+n p[p\right. \\
\quad+3] / 2+2 n^{2} \\
\quad-n / 3)\end{array}$ & $\begin{array}{c}J\left(5 n^{3} / 6+n p q / 2\right. \\
\quad+2 n^{2}-n / 3 \\
\quad+n / 2[p-q])\end{array}$ & $\begin{array}{c}J\left(5 n^{3} / 6+n p q / 2\right. \\
\quad+2 n^{2}-n / 3 \\
\quad-n / 2[p-q])\end{array}$ \\
\hline
\end{tabular}

equations for each mesh interval, but not interchanging equations for different intervals. The validity of this procedure was first proven by Mr. A. B. White [10], and we present a form of this basic result as the following theorem.

THEOREM. Let the block tridiagonal matrix $\mathbb{A}$ of $(5.4 \mathrm{~b}, \mathrm{c})$ be nonsingular. Then with appropriate row interchanges within each of the $n$ rows with indices $k$ in $j n+p<k \leqq(j+1) n+p$ for each $j=0,1, \cdots, J-1$, the factorization $\mathbb{A}$ $=\left[\beta_{j}, I, 0\right]\left[0, \alpha_{j}, C_{j}\right]$ is valid.

Proof. Suppose $\alpha_{k}$ has been computed for any $k=0,1, \cdots, J-1$. Since $\alpha_{0}=A_{0}$, we can start. Then we will show that:

(5.8a) the first $p$ rows of $\alpha_{k}$ are linearly independent,

$$
\operatorname{rank}\left(\begin{array}{c}
\alpha_{k} \\
B_{k+1}
\end{array}\right)=n \text {. }
$$

To do this, we define the square matrix of order $(J-k+1) n$ :

$$
\mathbb{A}_{k} \equiv\left(\begin{array}{cccc}
\alpha_{k} & C_{k} & & 0 \\
B_{k+1} & A_{k+1} & C_{k+1} & \\
C_{B_{J-1}} & A_{J}
\end{array}\right) .
$$

By the reduction procedure up to this stage, we must have

$$
\operatorname{det} \mathbb{A}=\operatorname{det} \alpha_{0} \cdots \operatorname{det} \alpha_{k-1} \operatorname{det} \mathbb{A}_{k} \neq 0 .
$$

If the first $p$ rows of $\alpha_{k}$ are linearly dependent, then $\mathbb{A}_{k}$ is singular (since the first $p$ rows of $C_{k}$ are zeros). Thus (5.8a) holds. On the other hand, if rank $\left(\begin{array}{c}\alpha_{k} \\ B_{k+1}\end{array}\right)<n$, then the first $n$ columns of $\mathbb{A}_{k}$ are linearly independent and again $\mathbb{A}_{k}$ is singular, so (5.8b) holds. 
To continue (or start if $k=0$ ) the reduction, we can perform row interchanges among the rows of index $p+1, \cdots, p+n$ in $\mathbb{A}_{k}$ to obtain a nonsingular matrix $\alpha_{k}$ in $(5.8 \mathrm{c})$. This follows from $(5.8 \mathrm{a}, \mathrm{b})$ and the fact that the last $q=n-p$ rows of $B_{k+1}$ are zeros. In these interchanges the zero patterns of $C_{k}, C_{k+1}$ and $B_{k+1}$ remain undisturbed, so the procedure can be continued.

Clearly in the final stage, $\alpha_{J}=\mathbb{A}_{J}$, and this must be nonsingular by (5.9). Thus the proof is complete.

It is of interest to note that while the first $p$ rows of $\alpha_{k}$ are being processed, for the Gauss eliminations used in $(5.6 \mathrm{c}, \mathrm{f})$, we can use column interchanges or row interchanges within the first $p$ rows; for the final $q$ rows, we switch over to row interchanges as in the theorem. With this mixed partial pivoting procedure, it is easily shown that the factorizations in cases (iii) and (iv) are valid under the hypothesis of the above theorem. Thus each of the factorizations indicated in (5.5) can be employed, with an appropriate restricted partial pivoting strategy, to solve the linear systems (5.3).

6. Practical considerations and an example. We point out that when part (iv) of the main theorem is applicable, Richardson extrapolation can be employed to get high accuracy with relatively few computations. Each extrapolation yields two orders of magnitude improvement in the numerical solution, provided that we can compute exact numerical solutions. Roundoff errors and iteration errors, of course, prevent this. However, if only $r-1$ extrapolations are to be made, so that approximations accurate to $O\left(h^{2 r}\right)$ are sought, then we can tolerate roundoff and iteration errors of this magnitude. Now since Newton's method converges quadratically, by part (ii) of the main theorem, it follows that the $v$ th iteration error will be $O\left(h^{2 v}\right)$ if the initial iterate, $U^{0}$, is within $O(h)$ of the exact numerical solution. Then we need perform only $v \approx \ln (2 r) / \ln (2)$ iterations to be consistent. For example, $v=3$ iterations suffice for $O\left(h^{8}\right)$ accuracy. When an accurate approximate solution has been obtained on the crudest net, this then furnishes, say by interpolation, an at least $O(h)$ approximation to the solution on the next refined net. If this procedure is continued, the above indicated theory is applicable, and we need only be concerned about the initial guess on the crudest net. There is no general theory available to insure an adequate determination of such initial estimates. Likewise, roundoff control may require double precision for very high accuracy, but, of course, the machine word length is crucial here.

The techniques indicated above have been applied with the box scheme (1.3), Newton's method (3.5) and case (i) block-factorization in (5.6) to solve many problems of scientific and technical interest. However, we show here the application to a simple example that has been treated by Jerome and Varga [2] using variational techniques with splines. The problem is:

$$
y^{\prime \prime}=e^{y},
$$

$$
y(0)=y(1)=0 .
$$

This has the unique solution

$$
y(t)=2 \ln \left\{C \sec \left[(C / 2)\left(t-\frac{1}{2}\right)\right]\right\}, \quad C=1.3360557 .
$$


Formulated as a first order system, $(6.1 \mathrm{a}, \mathrm{b})$ becomes

$$
\left\{\begin{array}{l}
y^{\prime}=z \\
z^{\prime}=e^{y}
\end{array},(1,0)\left(\begin{array}{l}
y(0) \\
z(0)
\end{array}\right)=0, \quad(1,0)\left(\begin{array}{l}
y(1) \\
z(1)
\end{array}\right)=0 .\right.
$$

We first employ the scheme (1.3) with Newton's method (3.5) on four nets with the uniform spacings $h=1 / 3,1 / 6,1 / 12,1 / 24$. Then since $y(t)$ and $z(t)$ are analytic (from (6.1c)), part (iv) of the main theorem holds and three extrapolations can be made on the initial net. The results of these extrapolations are indicated in Table 2, where the errors after the indicated extrapolations are shown (in standard floatingdecimal notation). Since the solution is symmetric about $x=1 / 2$, we only show the results for $y(1 / 3), z(1 / 3)$ and $z(0)$.

TABLE 2

Errors in computed solutions and in extrapolations

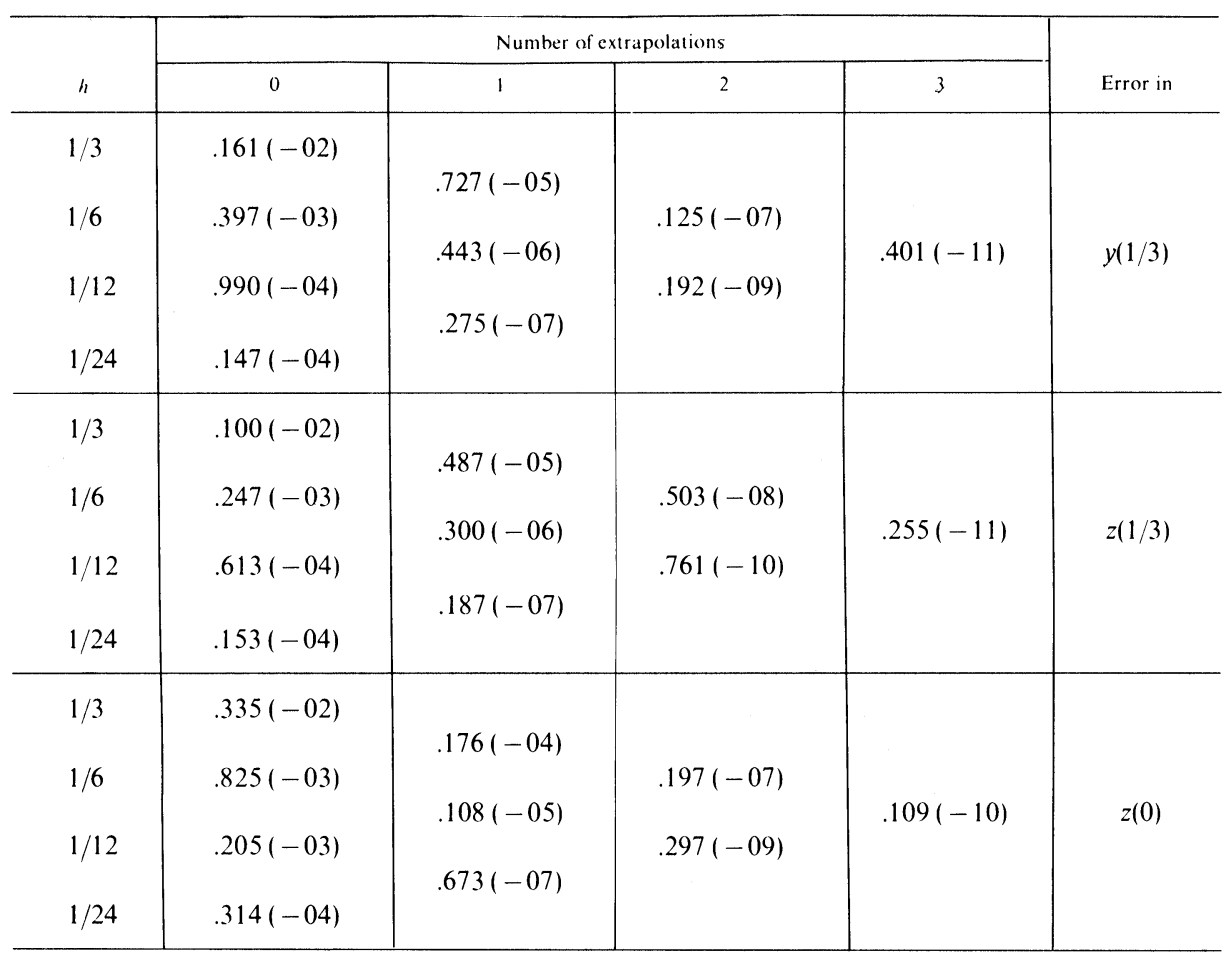

The convergence of the Newton iterates was very similar in all four cases. The worst case, for $h=1 / 12$, is reported in Table 3. The data for all initial iterates was

TABLE 3

Convergence of Newton iterates

\begin{tabular}{c|c|c}
\hline$v$ & $\max _{j}\left|y_{j}^{v}-y_{j}^{v-1}\right|$ & $\max _{j}\left|z_{j}^{v}-z_{j}^{v-1}\right|$ \\
\hline 1 & $.126(+01)$ & $.121(+01)$ \\
2 & $.786(-02)$ & $.572(-02)$ \\
3 & $.195(-06)$ & $.132(-06)$ \\
4 & $.206(-15)$ & $.241(-15)$ \\
\hline
\end{tabular}


chosen as $y_{j}^{0}=\left(t_{j}-\frac{1}{2}\right)^{2}-\frac{1}{4}$ and $z_{j}^{0}=2 t_{j}-1$. No doubt fewer iterates would have sufficed if we had bothered to use more accurate initial guesses (determined as previously indicated). All computations were done in double precision on the Caltech IBM 370/155. The program was most ably designed and employed by $\mathrm{Mr}$. S. A. Bolasna.

Note the high accuracy that can be obtained using relatively few net points; a total of 49 points was used on all the nets combined. From the first three nets, with a total of 24 points, we get absolute accuracy to within $2 \times 10^{-8}$, as is shown in Table 2. Also note that both the solution, $y(t)$, and its derivative, $z(t)$, are approximated to the same accuracy. These computations were repeated on other sets of nets, including several sets with nonuniform spacing, and very similar results were obtained. Jerome and Varga [2, Table II] get errors in $y(t)$ of at most 2.5 $\times 10^{-7}$ using natural cubic splines with only 10 nodes, that is, with spacing $h=1 / 9$. We cannot make further comparisons here, as the details of their solution of this problem are unknown to us.

Appendix. Multipoint boundary conditions. If the two-point boundary conditions $(1.1 \mathrm{~b})$ are replaced by the more general multipoint conditions :

$$
g\left(y\left(\tau_{1}\right), y\left(\tau_{2}\right), \cdots, y\left(\tau_{N}\right)\right)=0, \quad a \leqq \tau_{1}<\tau_{2}<\cdots<\tau_{N} \leqq b,
$$

then for isolated solutions of (1.1a), (A.1), we must replace (1.2b) by

$$
\sum_{v=1}^{N} B_{v} \phi\left(\tau_{v}\right)=0
$$

$$
B_{v} \equiv \frac{\partial}{\partial y\left(\tau_{v}\right)} g\left(y\left(\tau_{1}\right), \cdots, y\left(\tau_{N}\right)\right), \quad 1 \leqq v \leqq N .
$$

The net (1.3a) is required to be such that the boundary constraint points $\left\{\tau_{v}\right\}_{0}^{N}$ are contained in the net $\left\{t_{j}\right\}_{0}^{J}$, say,

$$
\tau_{v}=t_{j_{v}}, \quad 1 \leqq v \leqq N .
$$

The difference scheme is now (1.3b) and, replacing (1.3c), we see that

$$
g\left(u_{j_{1}}, u_{j_{2}}, \cdots, u_{j_{N}}\right)=0 .
$$

The main theorem remains valid with obvious modifications. In particular, now: (1.1a), (A.1) is to have an isolated solution;

$$
g\left(v_{1}, \cdots, v_{N}\right) \in C_{2}\left\{S_{\rho}\left[y\left(\tau_{1}\right)\right] \times \cdots \times S_{\rho}\left[y\left(\tau_{N}\right)\right]\right\} ;
$$

the difference equations (1.3a), (A.4) have a unique solution.

The analysis of $\S 2$ was originally carried out for the multipoint case in [2]. The changes required in $(2.1 \mathrm{~b}),(2.2),(2.4 \mathrm{~b})$ are obvious and lead to the replacement of $\mathbb{L}$ in $(2.6 \mathrm{c})$ by

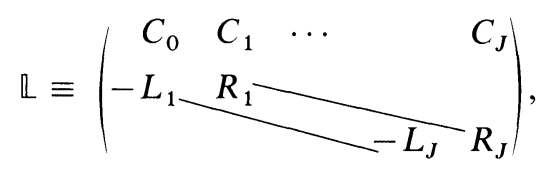




Now, the analysis of $\S 3$ remains valid after replacing $g\left(u_{0}, u_{j}\right)$ in (3.1c) by $g\left(u_{j_{1}}\right.$, $\left.u_{j_{2}}, \cdots, u_{j_{N}}\right)$, employing $\mathbb{L}$ as above, and requiring that $K$ be the maximum of the Lipschitz constants for $f_{y}(t, z), \partial g\left(v_{1}, \cdots, v_{N}\right) / \partial v_{v}$ with respect to $z, v_{v}, v=1,2, \cdots$, $N$.

The error estimates through (4.8), and hence part (iii) of the modified main theorem, require only using

(A.6a) $\quad \widetilde{B}_{v}=\int_{0}^{1} \frac{\partial g}{\partial y\left(\tau_{v}\right)}\left(y\left(\tau_{1}\right)+s v_{t_{1}}, \cdots, y\left(\tau_{N}\right)+s v_{t_{N}}\right) d s, \quad 1 \leqq v \leqq N$,

to replace $(4.1 \mathrm{~b})$ by

$$
\sum_{v=1}^{N} \widetilde{B}_{v} v_{t_{v}}=0
$$

The asymptotic error estimates require slightly more involved modifications. Of course, $(4.9 \mathrm{~b})$ is replaced by

$$
\sum_{s=1}^{N} B_{s} e^{v}\left(\tau_{s}\right)=\gamma_{v},
$$

and the family of nets $\left\{t_{k, j}\right\}_{0}^{J_{k}}, k=0,1,2, \cdots$, must each satisfy (A.3). The $\Theta_{v}(t)$ to be used in (4.9a) are unaltered and only the $\gamma_{v}$ for (A.7) need be changed. However, these are again easily obtained by formal Taylor expansions.

\section{REFERENCES}

[1] W. B. GragG, On extrapolation algorithms for ordinary initial value problems, this Journal, 2 (1965), pp. 384-403.

[2] J. W. JEROME AND R. S. VARGA, Generalizations of spline functions and applications to nonlinear boundary value and eigenvalue problems, Theory and Applications of Spline Functions, Academic Press, New York, 1969.

[3] H. B. KELLER, Accurate difference methods for linear ordinary differential systems subject to linear constraints, this Journal, 6 (1969), pp. 8-30.

[4] - Numerical Methods for Two-Point Boundary-Value Problems, Ginn-Blaisdell, Waltham, Mass., 1968.

[5] - Newton's method under mild differentiability conditions, J. Comput. System Sci., 4 (1970), pp. 15-28.

[6] H.-O. KreIss, Difference approximations for boundary and eigenvalue problems for ordinary differential equations, Math. Comp., 26 (1972), pp. 605-624.

[7] F. Nieuwstadt and H. B. Keller, Viscous flow past circular cylinders, Computers and Fluids, 1 (1973), pp. 59-71.

[8] J. Ortega AND W. Rheinboldt, Iterative Solution of Nonlinear Equations in Several Variables, Academic Press, New York, 1970.

[9] J. M. VARAH, On the solution of block tridiagonal systems arising from certain finite-difference equations, Math. Comp., 26 (1972), pp. 859-868.

[10] A. B. White, Ph.D. thesis, California Institute of Technology, Pasadena, Calif., in preparation.

[11] R. Weiss, The application of implicit Runge-Kutta and collocation methods to boundary value problems, to appear. 